\title{
Impact of Ethno-Cultural Beliefs On A Person With Mental Illness: A Case Report
}

\author{
Rajbhandari N1', Shakya DR², Sapkota N33, Basnet $\mathrm{M}^{4}$
}

\author{
1. Junior Resident 2. Additional Prof. 3. Asssoc. Prof. 4 Asst. Prof. Department of psychiatry, BPKIHS, Dharan, \\ Nepal
}

Email Corresponding Author: rajbhandarinikesh@gmail.com

\begin{abstract}
Culture, though difficult to define, is a collection of beliefs, attitudes, knowledge, customs, habits and behaviour which influence cognitions and social development of a patient. Cultures determine how sickness and illness are defined and that will determine what the first port of call is. It also colors the psychopathology. We here describe a case of a 36 years woman who presented with psychosis which co-occurred with left temporal lobe lesion who had significant delay in receiving proper treatment because of the ethno-cultural beliefs.
\end{abstract}

Keywords: Ethno-cultural Belief, mental illness, temporal lobe lesion

\section{INTRODUCTION}

Culture, though difficult to define, is a collection of beliefs, attitudes, knowledge, customs, habits and behaviour which influence cognitions and social development of a patient. ${ }^{1}$ Mental disorder is defined as behavioural or psychological syndrome or pattern associated with distress or significantly increased risk of suffering, pain or death. ${ }^{2}$ Culture defines deviance, how emotional distress is expressed and from where help is sought. Cultures determine how sickness and illness are defined and that will determine what the first port of call is. ${ }^{1}$ Relationship between culture and psychopathology was elaborated by Tseng (2003). ${ }^{3}$ Traditional healers are often the first port of help-seeking by caretakers. ${ }^{4}$ Witchcraft ideation is common in Nepal and many popular healers practice here, including the jhankri or spirit medium, the tantrik or healer practised in tantrism and the deuta, a healer possessed by a mother goddess, Hartimata. ${ }^{5}$ The spirit possession phenomenon is well recognised in Nepal, without underlying psychiatric illness. We here describe a case of a 36 years woman who presented with psychosis which co-occurred with left temporal lobe lesion.

\section{CASE REPORT:}

36 years housewife from Dharan, with no past history of psychiatric illness, was brought with complaints since 3 months of command auditory hallucinations with religious content this was followed by her refusing to eat, limiting herself to only drinking water since 2 months. In reaction to this hallucination she would also have trance and possession spells. She would also spend hours maintaining one single posture, refusing to move or to any efforts to move. Initially she was taken to different faith healers, but her behaviour continued to be the same. When her family was advised to seek medical help, they rejected, believing that she had been possessed by a 'MATA' (Deity) and was becoming a healer herself. After 3 months, patient became weak and possession spells stopped, she became bed-ridden, passed urine and stool in bed. On presentation to Emergency patient was in catatonia with mutism, stupor and negativism, though neurological examination could not be performed (due to negativism), other physical examination did not reveal any abnormalities. Patient was treated with intravenous. fluids, injections Lorazepam (for catatonia) and tablet Olanzapine $5 \mathrm{mg}$. Oral feeding improved from the 3rd day, patient started speaking from the 8th day and further examinations revealed neurological deficits with nystagmus, ptosis of the left eye, slurred speech and difficulty maintaining body balance. Magnetic Resonance Imaging of Brain (Fig 
1) revealed a small focus of altered signal intensity in left temporal lobe and a lesion close to atrium of ipsilateral ventrical showed few enhancing vessels close to it suggestive of ?Angioma. Patient was discharged after 15 days of admission. She was free of psychotic symptoms but had persisting neurological symptoms and so was referred to a neurologist. She had another MRI done (Fig 2) in a different centre which revealed a T2 hyperintense lesion with peripheral hypointensity lesion in left temporal lobe abutting the posterior aspect of the sylvian fissure near the temporal horn of lateral ventricle and the impression of cavernous angioma in the left temporal lobe was made.

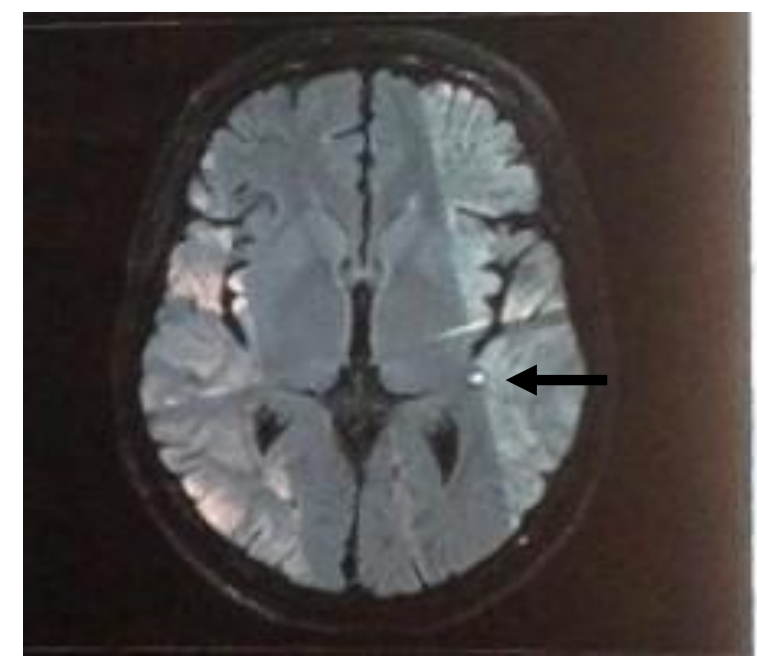

Figure1. Showing Magnetic Resonance Imaging of Brain revealed a small focus of altered signal intensity in left temporal lobe and a lesion close to atrium of ipsilateral ventrical showed few enhancing vessels close to it

\section{DISCUSSION:}

Patient presented with command hallucinations, hence she had psychosis. Neurological and MRI findings suggest that psychosis was due to brain pathology. Several cases of temporal lobe lesions associated with psychotic symptoms have been described. For example, Ortiz et $\mathrm{al}^{5}$ described a patient developing psychotic depression having acquired an ischemic left temporal lesion, Mizukami et $\mathrm{al}^{6}$ reported on a psychotic patient with a right temporal lesion of unknown origin, Blackshaw and Bowen ${ }^{7}$ presented a patient with atypical psychosis co-occurring with a left frontotemporal arachnoidal cyst.

It is known that most ethnic communities in Nepal have strong belief in traditional healing and traditional healers (locally known as baidangis, dhamis, jhankris, bijuwas, and dhami-jhankris). These healers are usually the first-contact for illness was shown in a study done in Dharan in 20094. In a descriptive cross-sectional study done in Dharan about $48 \%$ primary caretakers believed that performing magico-religious rituals could improve the behavior of the patients and $76 \%$ of them had visited faith healer for this purpose. ${ }^{9}$ Due to this Ethno-culutral beliefs our patient too had delay in receiving treatment, which eventually caused her to be bed-ridden.

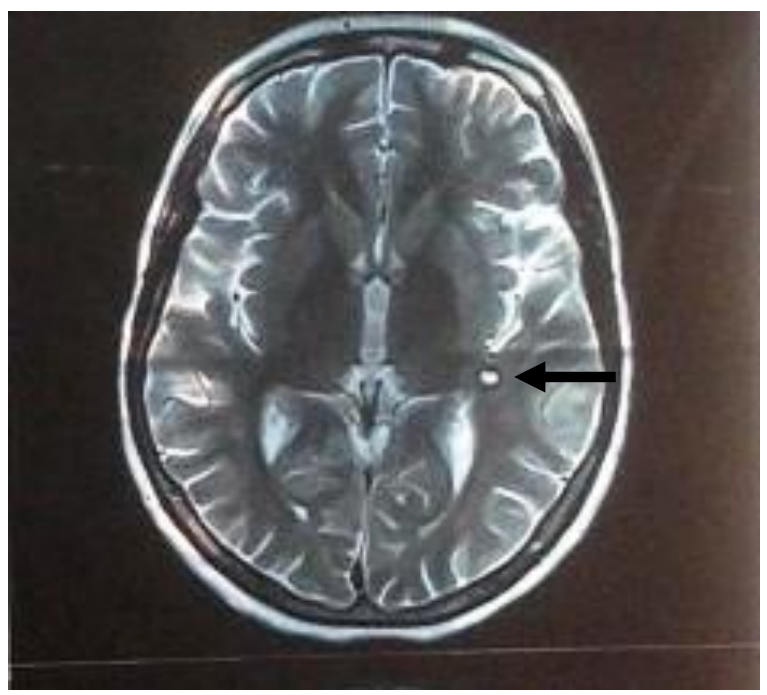

Figure 1. showing revealed a T2 hyperintense lesion with peripheral hypointensity lesion in left temporal lobe abutting the posterior aspect of the sylvian fissure near the temporal horn of lateral ventricle

\section{REFERENCES:}

1. Kalra G, Bhugra D, Shah N. 1Cultural aspects of schizophrenia. International Review of Psychiatry. October 2012; 24(5): 441-449.

2. American Psychiatric Association. (2000). Diagnostic and statistical manual of mental disorders (4thed., text rev.). Washington, DC: Author.

3. Tseng W.S . (2003). Clinician's Guide to Cultural Psychiatry. San Diego, CA: Academic Press .

4. Subedi S. Study of Knowledge, Attitude, Stigma and Pathway to care among patients with epilepsy and their primary caretakers. Unpublished (MD thesis). 2009.

5. Melia WMA, Mumford JD. Spirit Possession and Bewitchment Presenting as Physical Illness: Report of four Cases in Nepalese Males. I R Army Med Corps 1987;133:152-4

6. Ortiz N, Annoni JM, Trojan D, et al. Persistent severe depressive episode with mood-congruent psychotic features associated with left temporal ischemia. CognBehav Neurol. 2004;17:157-162.

7. Mizukami K, Yamakawa Y, Yokoyama $H$, et al. A case of psychotic disorder associated with a right temporal lesion: a special reference to magnetic resonance imaging and single photon emission 
computed tomography findings. Psychiatry ClinNeurosci. 1999;53:603-606.

8. Blackshaw S, Bowen RC. A case of atypical psychosis associated with alexithymia and a left fronto-temporal lesion: possible correlations. Can J Psychiatry. 1987;32:688-692.

9. Sapkota N, Pandey AK, Adhikari BR, Shyangwa PM, Shakya R. Magico-Religious beliefs among primary care takers of manic patients. I Psychiatrists' Association of Nepal. 2013;2(1): 7-13. 